\title{
CONJECTURED INEQUALITIES FOR JACOBI POLYNOMIALS AND THEIR LARGEST ZEROS
}

\author{
WALTER GAUTSCHI* AND PAUL LEOPARDI ${ }^{\dagger}$
}

\begin{abstract}
Inequalities are conjectured for the Jacobi polynomials $P_{n}^{(\alpha, \beta)}$ and their largest zeros. Special attention is given to the cases $\beta=\alpha-1$ and $\beta=\alpha$.
\end{abstract}

Key words. Jacobi polynomials, zeros, inequalities

AMS subject classifications. $33 \mathrm{C} 45$

1. Introduction. Special Jacobi polynomials $P_{n}^{(\alpha, \beta)}(x)$ with parameters $\beta=$ $\alpha-1$ or $\beta=\alpha$ are frequently encountered in multivariate polynomial approximation on spherical surfaces, in which case $\alpha$ is related to the space dimension; see, e.g., [4], $[5, \S 14.1]$. Technical properties, especially inequalities, for these polynomials can be a valuable aid in simplifying various estimates in the theory of spherical approximation. In this paper inequalities are studied related to the largest zeros of Jacobi polynomials and also inequalities involving the Jacobi polynomials themselves, more precisely, the scaled polynomials having the value 1 at $x=1$. All inequalities are only conjectured to hold, but compelling evidence is provided, both numerical and analytic, in support of their validity.

The special Jacobi polynomials with $\beta=\alpha-1$ are considered in $\S 2$, with inequalities for the largest zeros being discussed in $\S 2.1$, and inequalities for the scaled polynomials in $\S 2.2$. In $\S 3$, the analogous problems, and a variation thereof, for general Jacobi polynomials are taken up. Some special cases that can be proved rigorously are mentioned in $\S 4$.

\section{Special Jacobi polynomials.}

2.1. Largest zeros. Let $x_{n}^{(\alpha)}=\cos \Theta_{n}^{(\alpha)}, 0<\Theta_{n}^{(\alpha)}<\pi$, be the largest zero of the Jacobi polynomial $P_{n}^{(\alpha, \alpha-1)}(x), \alpha>0$. Our conjecture relates to the inequality

$$
n \Theta_{n}^{(\alpha)}<(n+1) \Theta_{n+1}^{(\alpha)} .
$$

From the interlacing property of the zeros of orthogonal polynomials it is known that the sequence $\left\{\Theta_{n}^{(\alpha)}\right\}$ is monotonically decreasing. Inequality (2.1), if true, places a limit on the relative decrement, $\left(\Theta_{n}^{(\alpha)}-\Theta_{n+1}^{(\alpha)}\right) / \Theta_{n+1}^{(\alpha)}<1 / n$.

Conjecture 1. Given $\alpha>0$, there are two alternatives: either (2.1) holds for all $n=1,2,3, \ldots$, or (2.1) is false for $n=1$. In other words, the validity of (2.1) for $n=1$ implies the validity of (2.1) for all $n \geq 1$.

Numerical evidence for Conjecture 1 was obtained with the help of the Matlab package OPQ available on the web site http://www.cs.purdue.edu/archives/2002/ wxg/codes. The following routine is at the core of the verification effort:

\footnotetext{
${ }^{*}$ Department of Computer Sciences, Purdue University, West Lafayette, Indiana 47907-2066 (wxg@cs.purdue.edu).

$\dagger$ School of Mathematics and Statistics, University of New South Wales, Sydney, NSW 2052 (leopardi@maths.unsw.edu.au).
} 


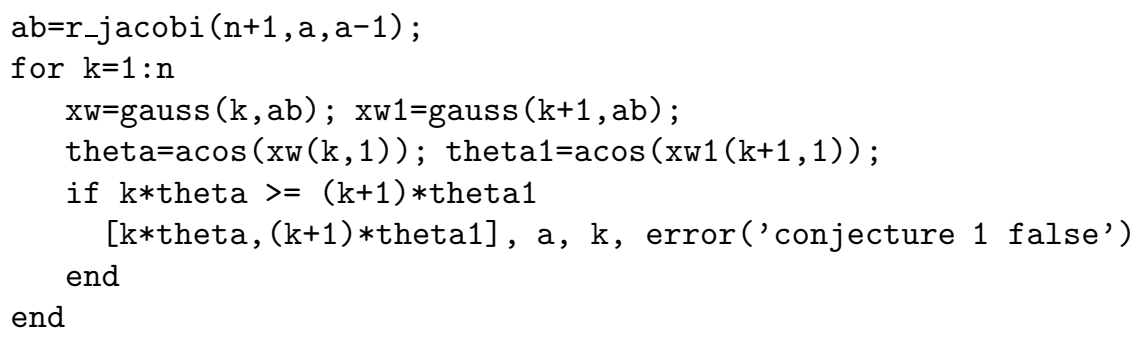

The first command generates the recursion coefficients for the special Jacobi polynomials, which are used in the routine gauss to compute the nodes and weights of the respective Gaussian quadrature rules. Only the nodes, stored (in increasing order) in the first column of the array xw resp. xw1 are of interest here.

When the verification routine is run with $\mathrm{n}=100$ and $\mathrm{a}=[0.5: 0.01: 1,1.1$ : $0.1: 10,10.5: 0.5: 20]$, the error statement is never invoked. On the other hand, when $\mathrm{a}=0.5:-0.01: 0.01$, the error message appears with $\mathrm{a}=0.13, \mathrm{n}=1$, and likewise, when $\mathrm{a}=0.14:-0.0001: 0.13$, it appears with $\mathrm{a}=0.1350, \mathrm{n}=1$. It thus appears that Conjecture 1 is true, and that inequality (2.1) holds for all $n \geq 1$ and for all $\alpha>\alpha_{0}$, where $0.1350<\alpha_{0}<0.1351$. In order to determine $\alpha_{0}$ more precisely, we examine the case $n=1$.

From the recurrence relation for Jacobi polynomials (see, e.g., [7, eqn (4.5.1)]) one finds

$$
\begin{gathered}
P_{1}^{(\alpha, \alpha-1)}(x)=\frac{1}{2}((2 \alpha+1) x+1), \\
4 P_{2}^{(\alpha, \alpha-1)}(x)=(\alpha+1)\left((2 \alpha+3) x^{2}+2 x-1\right) .
\end{gathered}
$$

Therefore,

$$
x_{1}^{(\alpha)}=-\frac{1}{2 \alpha+1}, \quad x_{2}^{(\alpha)}=\frac{1}{1+\sqrt{2 \alpha+4}},
$$

and (2.1) for $n=1$ is equivalent to

$$
\arccos \left(-\frac{1}{2 \alpha+1}\right)<2 \arccos \frac{1}{1+\sqrt{2 \alpha+4}},
$$

or, using $\arccos (-t)=\pi-\arccos (t)$, equivalent to

$$
2 \arccos \frac{1}{1+\sqrt{2 \alpha+4}}+\arccos \frac{1}{2 \alpha+1}-\pi>0 .
$$

The left-hand side is a strictly increasing function of $\alpha$, negative for $\alpha=0$ and tending to $\frac{1}{2} \pi$ as $\alpha \rightarrow \infty$. Therefore, if $\alpha_{0}$ is the unique root of

$$
2 \arccos \frac{1}{1+\sqrt{2 \alpha+4}}+\arccos \frac{1}{2 \alpha+1}-\pi=0,
$$

then (2.4), and hence (2.1) for $n=1$, holds exactly if $\alpha>\alpha_{0}$. Using the Matlab routine fzero, one finds

$$
\alpha_{0}=0.13507978085964 \text {. }
$$

Thus, if Conjecture 1 is true, then (2.1) holds for all $n \geq 1$ precisely if $\alpha>\alpha_{0}$. 
2.2. Scaled polynomials. For the remainder of this paper, we use the abbreviated notation

$$
\widetilde{P}_{n}^{(\alpha, \beta)}(x):=\frac{P_{n}^{(\alpha, \beta)}(x)}{P_{n}^{(\alpha, \beta)}(1)} .
$$

The conjecture for the Jacobi polynomials themselves involves the inequality

$$
\widetilde{P}_{n}^{(\alpha, \alpha-1)}\left(\cos \frac{\theta}{n}\right)<\widetilde{P}_{n+1}^{(\alpha, \alpha-1)}\left(\cos \frac{\theta}{n+1}\right) .
$$

With notation as in $\S 2.1$ we consider two intervals for $\theta$,

$$
0<\theta<\Theta_{1}^{(\alpha)}, \text { and } 0<\theta<\pi
$$

where

$$
\cos \Theta_{1}^{(\alpha)}=x_{1}^{(\alpha)}=-\frac{1}{2 \alpha+1} .
$$

Conjecture 2. Given $\alpha>0$, there are two alternatives for each of the two intervals (2.9): either (2.8) holds for all $n=1,2,3, \ldots$ and all $\theta$ in the respective interval, or (2.8) is false for $n=1$ and some $\theta$ in the respective interval. In other words, the validity of (2.8) for $n=1$ implies the validity of (2.8) for all $n \geq 1$.

The verification routine for Conjecture 2 is a bit more intricate than the one for Conjecture 1. Its core is shown below.

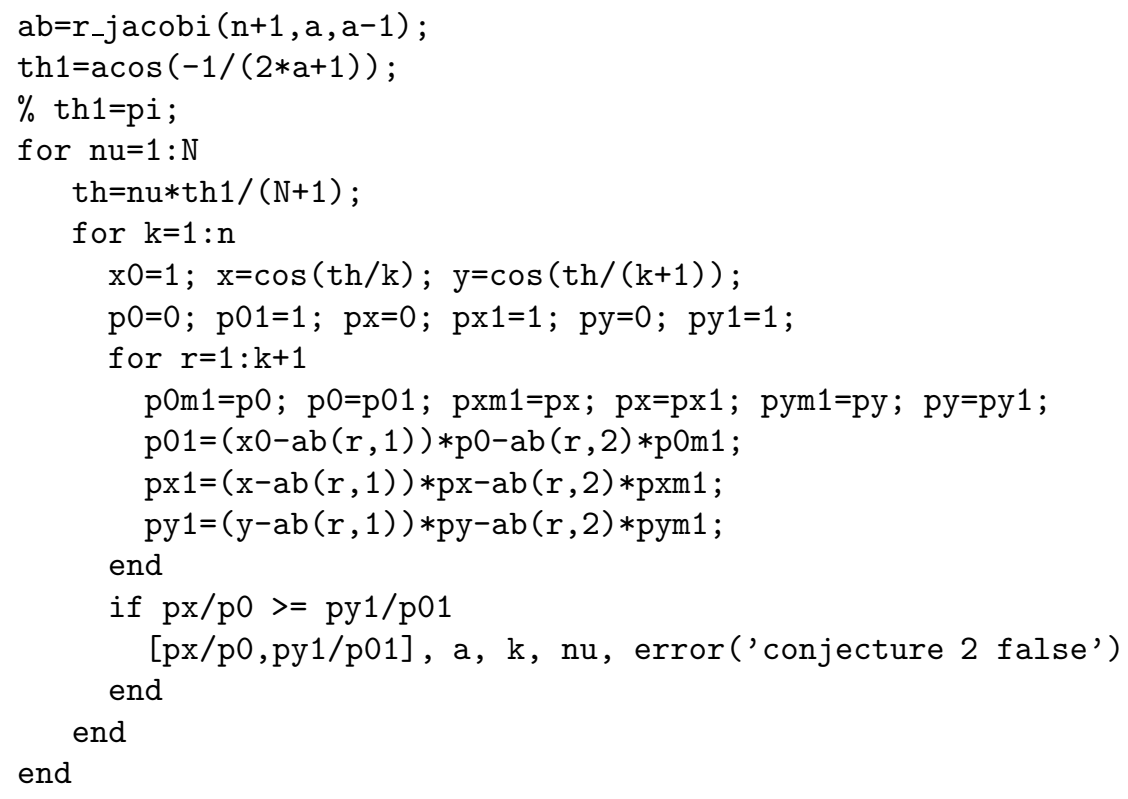

Run with $\mathrm{n}=100, \mathrm{~N}=1000$, and a as in $\S 2.1$, the routine for the first interval of (2.9) produces the same results as in $\S 2.1$, provided $\mathrm{N}$ is increased to $\mathrm{N}=5000$ for the last set of a-values. Conjecture 2 thus appears to be true, and inequality (2.8) valid for $0<\theta<\Theta_{1}^{(\alpha)}$ precisely if $\alpha>\alpha_{0}$. In the case of the second interval $0<\theta<\pi$, the first set of a-values, when $\mathrm{N}=1000$, again produces no error message, the second set, with $\mathrm{N}=5000$, an error message with $\mathrm{a}=0.28, \mathrm{n}=1$, and $\mathrm{a}=0.29:-0.001: 0.28$ 
an error message with $\mathrm{a}=0.280, \mathrm{n}=1$. Inequality (2.8) for the second interval thus seems to hold if $\alpha>\alpha_{1}$, where $0.280<\alpha_{1}<0.290$.

To get more precise information, we analyze the case $n=1$, i.e.,

$$
\widetilde{P}_{1}^{(\alpha, \alpha-1)}(\cos \theta)<\widetilde{P}_{2}^{(\alpha, \alpha-1)}\left(\cos \frac{\theta}{2}\right) .
$$

From (2.2), we have

$$
\begin{aligned}
& \widetilde{P}_{1}^{(\alpha, \alpha-1)}(\cos \theta)=\frac{(2 \alpha+1) \cos \theta+1}{2(\alpha+1)}, \\
& \widetilde{P}_{2}^{(\alpha, \alpha-1)}\left(\cos \frac{\theta}{2}\right)=\frac{(2 \alpha+3) \cos ^{2} \frac{\theta}{2}+2 \cos \frac{\theta}{2}-1}{2(\alpha+2)},
\end{aligned}
$$

so that (2.11), using $\cos \theta=2 \cos ^{2} \frac{\theta}{2}-1$, becomes

$$
\left(1+5 \alpha+2 \alpha^{2}\right) \cos ^{2} \frac{\theta}{2}-2(1+\alpha) \cos \frac{\theta}{2}+\left(1-3 \alpha-2 \alpha^{2}\right)<0,
$$

or, simplifying,

$$
(u-1)\left[\left(1+5 \alpha+2 \alpha^{2}\right) u-\left(1-3 \alpha-2 \alpha^{2}\right)\right]<0, \quad u:=\cos \frac{\theta}{2} .
$$

Since $u-1<0$ on either interval (2.9), this is the same as

$$
\left(1+5 \alpha+2 \alpha^{2}\right) u-\left(1-3 \alpha-2 \alpha^{2}\right)>0,
$$

or, since $1+5 \alpha+2 \alpha^{2}>0$,

$$
u>\frac{1-3 \alpha-2 \alpha^{2}}{1+5 \alpha+2 \alpha^{2}}, \quad u:=\cos \frac{\theta}{2} .
$$

Consider first the interval $0<\theta<\Theta_{1}^{(\alpha)}$. Then (2.13) holds precisely if

$$
\cos \frac{\Theta_{1}^{(\alpha)}}{2}=\sqrt{\frac{1+\cos \Theta_{1}^{(\alpha)}}{2}}=\sqrt{\frac{\alpha}{2 \alpha+1}}>\frac{1-3 \alpha-2 \alpha^{2}}{1+5 \alpha+2 \alpha^{2}} .
$$

Using the Matlab routine fzero, one finds

$$
\alpha>\alpha_{0}
$$

where, interestingly, $\alpha_{0}$ is exactly the same as in (2.6).

On the second interval $0<\theta<\pi$, we have (2.13) precisely if

$$
\cos \frac{\pi}{2}=0>\frac{1-3 \alpha-2 \alpha^{2}}{1+5 \alpha+2 \alpha^{2}}
$$

i.e., if

$$
\alpha>\alpha_{1}=\frac{1}{4}(\sqrt{17}-3)=.28077640640442 .
$$

In summary, if Conjecture 2 is true, then the inequality (2.8) holds for all $n \geq 1$ on the first interval (2.9) precisely if $\alpha>\alpha_{0}$, and on the second interval precisely if $\alpha>\alpha_{1}$, where $\alpha_{0}, \alpha_{1}$ are given by (2.6) and (2.16), respectively. 
We remark that by squaring (2.14) and removing the root $\alpha=-1$, one finds that $\alpha_{0}$ is the smallest positive root of the quartic equation

$$
4 \alpha^{4}+4 \alpha^{3}-11 \alpha^{2}-6 \alpha+1=0 .
$$

The same equation can be obtained from (2.5), written in the form

$$
2 \arccos \frac{1}{1+\sqrt{2 \alpha+4}}=\arccos \left(-\frac{1}{2 \alpha+1}\right) .
$$

Indeed, observing that $2 \arccos t=\arccos \left(2 t^{2}-1\right)$, eqn (2.18) implies

$$
\frac{2}{(1+\sqrt{2 \alpha+4})^{2}}-1=-\frac{1}{2 \alpha+1},
$$

or

$$
\alpha(1+\sqrt{2 \alpha+4})^{2}=2 \alpha+1 .
$$

By an elementary calculation, this yields (2.17).

\section{General Jacobi polynomials.}

3.1. Largest zeros. We now denote by $x_{n}^{(\alpha, \beta)}=\cos \Theta_{n}^{(\alpha, \beta)}, 0<\Theta_{n}^{(\alpha, \beta)}<\pi$, the largest zero of the Jacobi polynomial $P_{n}^{(\alpha, \beta)}(x), \alpha>-1, \beta>-1$. We consider the inequality analogous to (2.1),

$$
n \Theta_{n}^{(\alpha, \beta)}<(n+1) \Theta_{n+1}^{(\alpha, \beta)} .
$$

The case $\alpha=\beta=-1 / 2$ of Chebyshev polynomials is exceptional here, since $\Theta_{n}=$ $\pi / 2 n$, and both sides of (3.1) are identically equal to $\pi / 2$.

Using an obvious extension of the Matlab routine in $\S 2.1$, we are led to conjecture:

Conjecture 3. Given $\alpha>-1, \beta>-1$, there are two alternatives: either (3.1) holds for all $n=1,2,3, \ldots$, or (3.1) is false for $n=1$. In other words, the validity of (3.1) for $n=1$ implies the validity of (3.1) for all $n \geq 1$.

It is known that

$$
\lim _{n \rightarrow \infty} n \Theta_{n}^{(\alpha, \beta)}=j_{1}^{(\alpha)},
$$

where $j_{1}^{(\alpha)}$ is the first positive zero of the Bessel function $J_{\alpha}$ (cf. [7, Theorem 8.1.2]). Conjecture 3 , if true, then states that the validity of (3.1) for $n=1$ implies that convergence in (3.2) is monotone increasing.

The following is our evidence for Conjecture 3. Running the (extended) verification routine of $\S 2.1$ with $n$ up to 100 , and for each $\alpha=1.01: 0.01: 1.2,1.3: 0.1: 5.0,6$ : $1: 20$ for $\beta=-0.99: 0.01:-0.80,-0.7: 0.1: 0.0,1: 1: 20$, no error message was encountered, suggesting that the inequality (3.1) holds for all $n \geq 1$ in the infinite domain $\alpha>1, \beta>-1$. When one takes $\alpha=0.9:-0.1:-0.9$, however, and for each of these $\alpha$ goes through $\beta=20:-1: 0,-0.1:-0.1:-0.9,-0.89:-0.01:-0.99,-0.999$, then an error message appears, always with $\mathrm{n}=1$, for the following pairs of values $(\alpha, \beta)$ :

\begin{tabular}{c|ccccccccccc}
$\alpha$ & 0.9 & 0.8 & 0.7 & 0.6 & 0.5 & 0.4 & 0.3 & 0.2 & 0.1 & 0 \\
\hline$\beta$ & -0.999 & -0.999 & -0.99 & -0.98 & -0.97 & -0.95 & -0.92 & -0.90 & -0.9 & -0.9 \\
& $\alpha$ & -0.1 & -0.2 & -0.3 & -0.4 & -0.5 & -0.6 & -0.7 & -0.8 & -0.9 & \\
\cline { 2 - 8 } & $\beta$ & -0.8 & -0.8 & -0.7 & -0.6 & -0.5 & -0.5 & -0.4 & -0.3 & -0.2 &
\end{tabular}


The results suggest that in the strip $-1<\alpha<1, \beta>-1$, there exists a curve, monotonically decreasing from 0 to -1 , above which (3.1) holds for all $n \geq 1$, and below which inequality (3.1) fails for $n=1$. We will compute this curve more accurately when, as we now begin to do, the case $n=1$ is examined.

In analogy to $(2.2)$, we find

$$
\begin{gathered}
P_{1}^{(\alpha, \beta)}(x)=\frac{1}{2}((\alpha+\beta+2) x+\alpha-\beta), \\
8 P_{2}^{(\alpha, \beta)}(x)=(\alpha+\beta+3)(\alpha+\beta+4) x^{2}+2(\alpha+\beta+3)(\alpha-\beta) x \\
+(\alpha-\beta)^{2}-(\alpha+\beta+4),
\end{gathered}
$$

from which

$$
\begin{aligned}
& x_{1}^{(\alpha, \beta)}=-\frac{\alpha-\beta}{\alpha+\beta+2}, \\
& x_{2}^{(\alpha, \beta)}=\frac{1}{\alpha+\beta+4}\left[-(\alpha-\beta)+2 \sqrt{2+\frac{\alpha \beta-2}{\alpha+\beta+3}}\right] .
\end{aligned}
$$

Inequality (3.1), therefore, analogously to (2.5), can be given the form

$$
\begin{gathered}
2 \arccos \left(\frac{1}{\alpha+\beta+4}\left[-(\alpha-\beta)+2 \sqrt{2+\frac{\alpha \beta-2}{\alpha+\beta+3}}\right]\right) \\
+\arccos \frac{\alpha-\beta}{\alpha+\beta+2}-\pi>0 .
\end{gathered}
$$

When $\alpha=\beta=-\frac{1}{2}$, this gives $2 \frac{\pi}{4}+\frac{\pi}{2}-\pi=0$, i.e., equality in (3.1), as was already noted above. The same is true for $\alpha=1$ and $\beta \rightarrow-1$, and for $\alpha>1$ and $\beta \rightarrow \infty$. When $\alpha>1$ is fixed, and $\beta$ increases from -1 to $\infty$, the graph of (3.5) sharply increases from a positive value to a maximum and then decreases monotonically to zero, so that (3.5) holds for all $\alpha>1, \beta>-1$, in agreement with what was found numerically above.

When $-1<\alpha<1$ is fixed, the equation in $\beta$ resulting from replacing inequality in (3.5) by equality, can be solved numerically by the Matlab routine fzero. This produces the curve shown in Fig 1. Inequality (3.1) for $n=1$ thus holds in the region

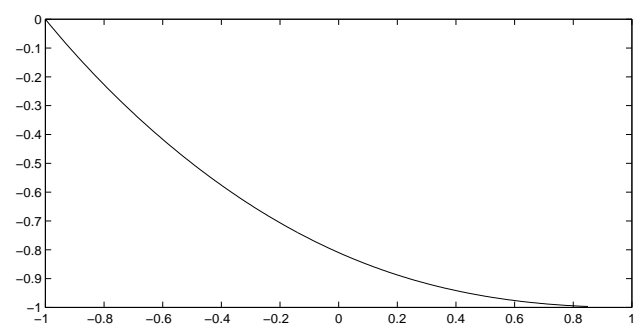

Figure 1. The boundary curve of the domain of validity for (3.1)

above this curve, and, together with $(-1<\alpha<1, \beta>0) \cup(1<\alpha<\infty, \beta>-1)$, this is the region of validity of the inequality for all $n \geq 1$ if Conjecture 3 is true. 
The graph also sheds new light on the result found in $\S 2.1$ : If the inequality is to be true for $(\alpha, \beta=\alpha-1)$, then the point of intersection of the line $\beta=\alpha-1$ with the boundary curve of Fig. 1 determines $\alpha_{0}$. Setting $\beta=\alpha-1$ in (3.5) and replacing the inequality sign with the equality sign indeed yields (2.5). Similarly, if $\beta=\alpha$, the point of intersection of the line $\beta=\alpha$ with the curve yields $\alpha=-\frac{1}{2}$, as is easily verified. Inequality (3.5) thus holds for $\beta=\alpha>-\frac{1}{2}$, and therefore, if Conjecture 3 is true, inequality (3.1) in the ultraspherical case $\beta=\alpha$ holds for all $\alpha>-\frac{1}{2}$. It is actually known to hold for $-\frac{1}{2}<\alpha<\frac{1}{2}$ in the sharper form $\left(n+\alpha+\frac{1}{2}\right) \Theta_{n}<\left(n+\alpha+\frac{3}{2}\right) \Theta_{n+1}$; cf. [7, $\S 6.3(5)$, p. 127]. However, this sharper inequality ceases to hold when $\alpha \geq \frac{1}{2}$.

3.2. Scaled polynomials. The inequality to be studied here is

$$
\widetilde{P}_{n}^{(\alpha, \beta)}\left(\cos \frac{\theta}{n}\right)<\widetilde{P}_{n+1}^{(\alpha, \beta)}\left(\cos \frac{\theta}{n+1}\right),
$$

with $\widetilde{P}$ defined by (2.7), on either of the two intervals

$$
0<\theta<\Theta_{1}^{(\alpha, \beta)}, \quad 0<\theta<\pi,
$$

where

$$
\cos \Theta_{1}^{(\alpha, \beta)}=x_{1}^{(\alpha, \beta)}=-\frac{\alpha-\beta}{\alpha+\beta+2} .
$$

We note again the exceptional case $\alpha=\beta=-1 / 2$, in which both sides of (3.6) are identically equal to $\cos \theta$.

Conjecture 4. Given $\alpha>-1, \beta>-1$, there are two alternatives for each of the two intervals (3.7): either (3.6) holds for all $n=1,2,3, \ldots$ and all $\theta$ in the respective interval, or (3.6) is false for $n=1$ and some $\theta$ in the respective interval. In other words, the validity of (3.6) for $n=1$ implies the validity of (3.6) for all $n \geq 1$.

Since

$$
P_{n}^{(\alpha, \beta)}(1)=\left(\begin{array}{c}
n+\alpha \\
n
\end{array}\right) \sim \frac{n^{\alpha}}{\Gamma(\alpha+1)} \text { as } n \rightarrow \infty,
$$

the result in [7, Theorem 8.1.1] can be rephrased in the form

$$
\lim _{n \rightarrow \infty} \widetilde{P}_{n}^{(\alpha, \beta)}\left(\cos \frac{\theta}{n}\right)=\Gamma(\alpha+1)\left(\frac{\theta}{2}\right)^{-\alpha} J_{\alpha}(\theta),
$$

where $J_{\alpha}$ is the Bessel function of order $\alpha$. Therefore, Conjecture 4 , if true, states that the validity of (3.6) for $n=1$ implies that convergence in (3.9) is monotone increasing.

The Matlab script of $\S 2.2$ is easily adapted to deal with the conjecture (3.6) for general Jacobi polynomials. When run with the same data as used to verify Conjecture 3 , with $n=100$ and $N=1000$, similar results were obtained as in $\S 3.1$, i.e., a strong indication that (3.6) holds on either interval (3.7) for all $n \geq 1$ whenever $\alpha>1$ and $\beta>-1$, while for $\alpha$ in the interval $(-1,1)$ the same is true for $\beta$ above a certain curve that extends from the point $(\alpha, \beta)=(-1,0)$ down to the point $(\alpha, \beta)=(1,-1)$. For $\beta$ below that curve, the conjecture fails consistently when $n=1$. As will be seen, the initial part of this curve, for $-1<\alpha<-\frac{1}{2}$, is the straight line $\beta=-\alpha-1$.

This all will become more clear by analyzing (3.6) in the case $n=1$,

$$
\widetilde{P}_{1}^{(\alpha, \beta)}(\cos \theta)<\widetilde{P}_{2}^{(\alpha, \beta)}\left(\cos \frac{\theta}{2}\right) .
$$


From (3.3) we first note that

$$
\widetilde{P}_{1}^{(\alpha, \beta)}(\cos \theta)=\frac{(\alpha+\beta+2) \cos \theta+\alpha-\beta}{2(\alpha+1)}
$$

and

$$
\widetilde{P}_{2}^{(\alpha, \beta)}\left(\cos \frac{\theta}{2}\right)=\frac{N(\alpha, \theta)}{4(\alpha+1)(\alpha+2)}
$$

where

$N(\alpha, \theta)=(\alpha+\beta+3)(\alpha+\beta+4) \cos ^{2} \frac{\theta}{2}+2(\alpha+\beta+3)(\alpha-\beta) \cos \frac{\theta}{2}+(\alpha-\beta)^{2}-(\alpha+\beta+4)$.

The inequality (3.10) then becomes, after simplification,

$$
(u-1)\left[\left(3 \alpha^{2}+2 \alpha \beta+9 \alpha-\beta^{2}+\beta+4\right) u+\alpha^{2}+2 \alpha \beta+\beta^{2}+3 \alpha+7 \beta+4\right]<0,
$$

with $u$ as in (2.13). Again, since $u-1<0$ on either of the two intervals (3.7), the inequality to be studied is

$$
\begin{gathered}
\left(3 \alpha^{2}+2 \alpha \beta+9 \alpha-\beta^{2}+\beta+4\right) u+\alpha^{2}+2 \alpha \beta+\beta^{2}+3 \alpha+7 \beta+4>0, \\
u:=\cos \frac{\theta}{2} .
\end{gathered}
$$

Lemma 3.1. Let $a, b$ be real numbers, and consider the inequality

$$
a u+b>0 \quad \text { on } u_{0}<u<1, u_{0} \geq 0 .
$$

If $a+b \geq 0$, then (3.12) is always true except when $a=b=0$ or $a>0, b<0$, and $u_{0}<-b / a$. If $a+b<0$, then (3.12) is never true.

Proof. Immediate on geometric grounds. $\mathrm{C}$

We now apply Lemma 3.1 to (3.11), i.e., to

$$
\begin{aligned}
& a=3 \alpha^{2}+2 \alpha \beta+9 \alpha-\beta^{2}+\beta+4, \\
& b=\alpha^{2}+2 \alpha \beta+\beta^{2}+3 \alpha+7 \beta+4 .
\end{aligned}
$$

Here, one computes

$$
a+b=4(\alpha+2)(\alpha+\beta+1) .
$$

Since $\alpha+2>0$, inequality (3.11) is false on either of the two intervals (3.7) if $\alpha+\beta+1<0$. In the case $\alpha+\beta+1 \geq 0$ it is false if $a=b=0$, which implies $\alpha=\beta=-\frac{1}{2}$, or if

$$
a>0, b<0 \text {, and } u_{0}<-b / a,
$$

with $a, b$ as defined in (3.13). The curve $b=0$ is given by

$$
\beta=-\alpha-\frac{7}{2}+\frac{1}{2} \sqrt{16 \alpha+33}, \quad-1<\alpha<1 .
$$

By plotting the respective curves in the $(\alpha, \beta)$-plane, one finds that $b<0$ combined with $\alpha+\beta+1 \geq 0$ and $\beta \geq-1$, cuts out the domain $D$ shown in Fig. 2. Inequality (3.11) thus holds for all $(\alpha, \beta)$ located above the upper boundary curve of $D$ and to 


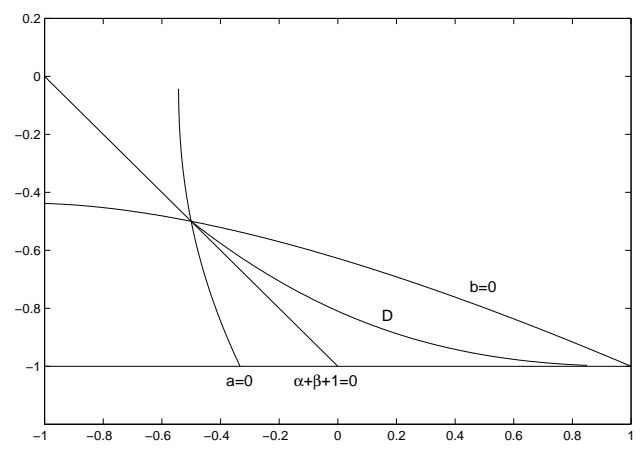

Figure 2. The boundary curves for the domain of validity of (3.10)

the right of the line $\alpha+\beta+1=0$, and for those $(\alpha, \beta)$ in the interior of $D$ precisely if $u_{0}>-b / a$ in (3.14). On the first interval (3.7), this will be true precisely if

$$
\begin{array}{r}
u_{0}=\cos \frac{\Theta_{1}^{(\alpha, \beta)}}{2}=\sqrt{\frac{1+\cos \Theta_{1}^{(\alpha, \beta)}}{2}}=\sqrt{\frac{\beta+1}{\alpha+\beta+2}} \\
>-\frac{\alpha^{2}+2 \alpha \beta+\beta^{2}+3 \alpha+7 \beta+4}{3 \alpha^{2}+2 \alpha \beta+9 \alpha-\beta^{2}+\beta+4} .
\end{array}
$$

This is the curve plotted inside the domain $D$ of Fig. 2, above which inequality (3.11) is true, and below which it is false. This, together with the discussion above, completely delineates the domain of validity of (3.10) on the first interval (3.7). On the second interval we have $u_{0}=\cos \frac{\pi}{2}=0$, and the third inequality in (3.14) is a consequence of the other two. Thus, (3.10) is false in all of $D$, and the domain of validity of (3.10) is the region above the upper boundary of $D$, to the right of the line $\alpha+\beta+1=0$, and of course bounded by the lines $\alpha=-1$ and $\beta=-1$. If Conjecture 4 is true, the same domains of validity hold for the inequality (3.6).

We remark that the special case $\beta=\alpha-1, \alpha>0$, turns (3.15) into (2.14), and the inequality $b>0$ into (2.16). Likewise, the line $\beta=\alpha, \alpha>-1$, passes through the point $\left(-\frac{1}{2},-\frac{1}{2}\right)$ where all the curves in Fig. 2 intersect. Consequently, (3.10), and if Conjecture 4 is valid, (3.6), is true for all $\beta=\alpha>-\frac{1}{2}$. S. Koumandos [2], in fact, has shown that (3.6) is true whenever $|\alpha|=|\beta|=\frac{1}{2}$ except for $\alpha=\beta=-\frac{1}{2}$.

In order to lend still more credence to the validity of Conjecture 4 , we ran the (extended) Matlab routine of $\S 2.2$ with $(\alpha, \beta)$ slightly above and below (at a distance of .01 from) the boundary curves of the domain of validity for (3.10). As expected, no error message appeared when $(\alpha, \beta)$ is above the boundary curve, and error messages consistently with $\mathrm{n}=1$ otherwise. (Only in the case of $u_{0}=0$, the maximum value of $n$ had to be lowered to $n=50$ to obtain sufficient numerical resolution along the straight part of the boundary curve.)

3.3. An alternative conjecture. Examination of the graphs of $\widetilde{P}_{n}^{(\alpha, \beta)}\left(\cos \frac{\theta}{n}\right)$ for numerous values of $\alpha, \beta$ and $n$ suggests that Conjectures 3 and 4 can be combined into the following conjecture.

Conjecture 5. Given $\alpha>-1, \beta>-1$, if (3.6) holds for $n=1$ and $0<\theta \leqslant$ $\Theta_{1}^{(\alpha, \beta)}$, then (3.6) holds for $0<\theta \leqslant n \Theta_{n}^{(\alpha, \beta)}$ for all $n=1,2,3, \ldots$. 
If (3.6) holds for $\theta=n \Theta_{n}^{(\alpha, \beta)}$ then we have

$$
\widetilde{P}_{n+1}^{(\alpha, \beta)}\left(\cos \frac{n \Theta_{n}}{n+1}\right)>0=\widetilde{P}_{n+1}^{(\alpha, \beta)}\left(\cos \Theta_{n+1}\right)
$$

and therefore $n \Theta_{n}^{(\alpha, \beta)}<(n+1) \Theta_{n+1}^{(\alpha, \beta)}$. In other words, if the premise of Conjecture 5 is true, then the conjecture implies (3.1).

To gain confidence in Conjecture 5, the verification routine of $\S 2.1$ was further modified. Following is the core of the Matlab routine used to verify Conjecture 5 .

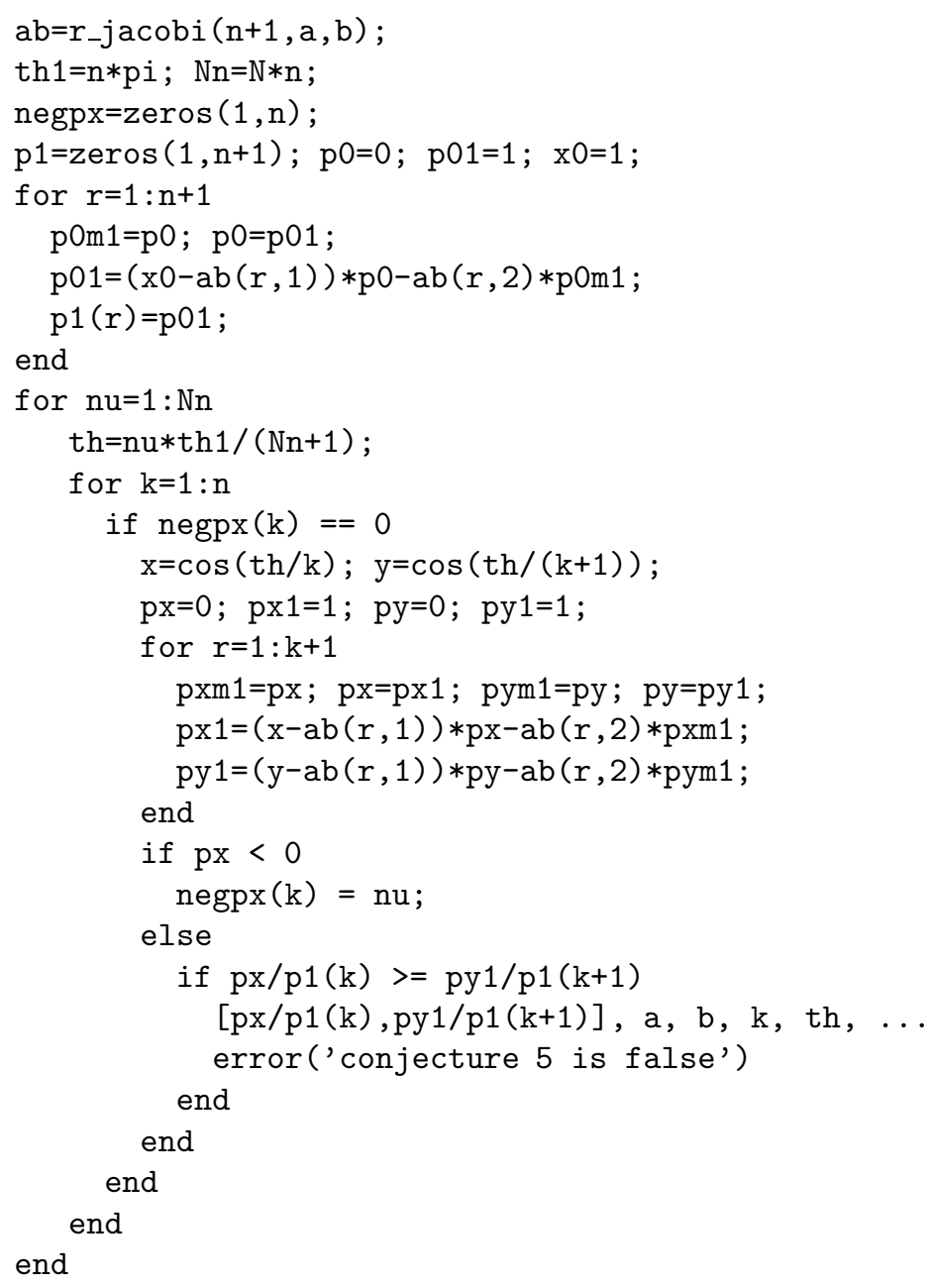

(To avoid overflow when $\alpha+\beta+2>128$, the statement defining mu in the routine

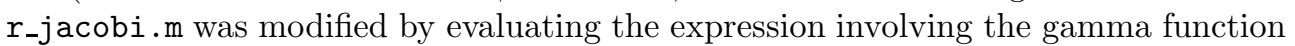
by first taking its logarithm and then exponentiating the result.)

This routine was run with $\mathrm{N}=15, \mathrm{n}=128$ and the following values of $\mathrm{a}$ and $\mathrm{b}$ :

1. $\mathrm{a}=2^{\mu}-1, \mathrm{~b}=2^{\nu}-1$, with $\mu, \nu \in\{-1,-0.9, \ldots, 6\}$,

2. $\mathrm{a} \in\{-0.95,-0.9, \ldots,-0.55\}, \mathrm{b}=2^{\nu}-1$, with $\nu \in\{-1,-0.9, \ldots, 6\}$, subject to $\mathrm{a}+\mathrm{b}+1>0$,

$3 . \mathrm{b} \in\{-0.95,-0.9, \ldots,-0.55\}, \mathrm{a}=2^{\mu}-1$, with $\mu \in\{1,1.1, \ldots, 6\}$, 
4. $\mathrm{b} \in\{-0.95,-0.9, \ldots,-0.55\}, \mathrm{a}=2^{\mu}-1$, with $\mu \in\{-1,-0.9, \ldots, 0.9\}$, subject to $\mathrm{a}=\alpha, \mathrm{b}=\beta$, such that $\alpha+\beta+1>0, \beta<-\alpha-\frac{7}{2}+\frac{1}{2} \sqrt{16 \alpha+33}$ and (3.15) holds.

In all cases, the error message was not seen.

4. Partial results. Apart from the result of Szegö $[7, \S 6.3(5)$, p. 127] and Koumandos [2], referred to above, the inequalities (3.1) and (3.6) are so far known to hold in only a few cases.

For the case where $(\alpha, \beta)$ lies in the square $\left(-\frac{1}{2}, \frac{1}{2}\right)^{2}$, the inequality $(3.1)$ can be proven for $n \geqslant 2$ either as a result of the inequalities of Gatteschi [1, Theorem 1.5, p. 1550], or directly using a version of the Sturm comparison theorem as formulated by Szegö [6, p. 3].

The paper [3] uses a different formulation of the Sturm comparison theorem to show that (3.6) holds for $n \geqslant 1, \alpha \geqslant \beta>-\frac{1}{2}, 0<\theta \leqslant \frac{\pi}{2}$.

\section{REFERENCES}

[1] Gatteschi, Luigi, New inequalities for the zeros of Jacobi polynomials, SIAM J. Math. Anal. 18 (1987), 1549-1562.

[2] Koumandos, Stamatis, Personal communication, 2005.

[3] Leopardi, Paul, Positive weight quadrature on the sphere and monotonicities of Jacobi polynomials, Numer. Algorithms (this issue).

[4] Reimer, Manfred, Hyperinterpolation on the sphere at the minimal projection order, $J$. Approx. Theory 104 (2000), 272-286.

[5] Reimer, Manfred, Multivariate polynomial approximation, Internat. Ser. Numer. Math., Vol. 144, Birkhäuser, Basel, 2003.

[6] Szegö, Gabriel, Inequalities for the zeros of Legendre polynomials and related functions, Trans. Amer. Math. Soc. 39 (1936), 1-17.

[7] Szegö, Gabor, Orthogonal polynomials, Colloquium Publications, Vol. 23, 4th ed., Amer. Math. Soc., Providence, RI, 1975. 